\title{
Analysis of Hydrocarbon Dendrimers by Laser Desorption Time-of-Flight and Fourier Transform Mass Spectrometry
}

\author{
Kathleen L. Walker, Michael S. Kahr, and Charles L. Wilkins \\ Department of Chemistry, University of California, Riverside, Riverside, California, USA \\ Zhifu $\mathrm{Xu}^{*}$ and Jeffrey S. Moore \\ Roger Adams Laboratory, Department of Chemistry, University of Illinois, Urbana, Urbana, Illinois, USA
}

\begin{abstract}
The first mass spectrometric analysis of a new class of hydrocarbon dendrimers that result from a convergent synthetic approach is reported. Molecular weights of a series of phenylacetylene dendrimers ( 715 to $14776 \mathrm{u} \mathrm{MW)}$ are characterized by ultraviolet matrix-assisted laser desorption (MALD) time-of-flight (TOF) mass spectrometry, direct and silver chemical ionization infrared laser desorption Fourier transform mass spectrometry (FTMS), and ultraviolet matrix-assisted laser desorption silver chemical ionization Fourier transform mass spectrometry. New matrices and techniques were developed to facilitate analysis of the dendrimers. Mass measurement accuracies between 10 and $25 \mathrm{ppm}$ are obtained for molecular ion species of the five dendrimers analyzed. Laser desorption time-of-flight and FTMS techniques are shown to be complementary, with FTMS providing high mass resolution (27,000-67,000 resolving power) and accuracy for lower mass dendrimers (10-14 ppm) and MALD TOF yielding the highest resolution (1100 resolving power) and accuracy (25 ppm) for the largest dendrimer. These results are consistent with proposed empirical formulas. ( $\mathrm{Am}$ Soc Mass Spectrom 1994, 5, 731-739)
\end{abstract}

\section{L} aser desorption mass spectrometry of hydrocarbon polymers and dendrimers has not been extensively investigated to date because such materials are less amenable to chemical ionization and matrix-assisted laser desorption-ionization (MALDI) than corresponding heteroatom-containing compounds. As a consequence, it is of particular interest to develop methods that will make it possible to use laser desorption (LD) techniques for hydrocarbon characterization with the same generality already available for polar polymers and dendrimers. Here, the use of matrix-assisted laser desorption-ionization time-offlight (TOF) and Fourier transform mass spectrometry (FTMS) for characterization of a group of phenylacetylene dendritic molecules is reported. These materials are of interest as models of molecular nanostructures that can be used for the study of physical properties as a function of molecular size and archilecture. Dendritic macromolecules also have potential applications in systems that exploit host-guest chemistry (such as drug delivery).

The synthesis of the phenyl acetylene dendrimers that are the focus of the present study was reported by

Address reprint requests to Charles L. Wilkins, Department of Chem-
istry, University of California, Riverside, Riverside, CA 92521.
"Present address; PPG Industries, Inc, Advanced Research Depart-
ment, Research Center, 4325 Rosanna Drive, Allison Park, PA 15101.
Xu et al. [1]. They used a convergent synthetic method that proceeds by assembling phenyl acetylene monodendrons around a trifunctional core. The zeroth tridendron is referred to as D-4, the first dendritic generation as D-10, the next as D-22, and so on. In each case, the numeric designation refers to the number of monomer units expected to be incorporated. Graphs of the molecular structures of the compounds studied here are contained in Figure 1. Figure 2 shows threedimensional representations of models of the D-22 and D-94 dendrimers. In the article cited [1], it was proposed that the synthetic route should result in very rigid dendritic structures that should have limited degrees of rotational freedom and that use of the convergent method would produce highly homogeneous products. Thus, mass spectrometry of the synthetic materials is useful both to assess homogeneity and, by accurate mass measurements, to confirm compositions. Size exclusion chromatography (SEC) with polystyrene standards failed to differentiate siliconcontaining synthetic intermediates from products, and the measurements were of sufficiently low resolution and accuracy that they could not be used for definitive mass determinations [1].

Laser desorption mass spectrometry is an attractive alternative for mass analysis of the phenyl acetylene dendrimers. It can provide highly accurate mass determinations for intact molecular ions of nonvolatile and 


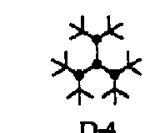

generation $n=0$

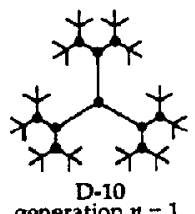

generation $n=1$

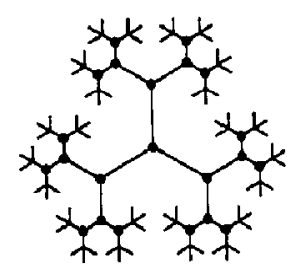
D-22 $n=2$

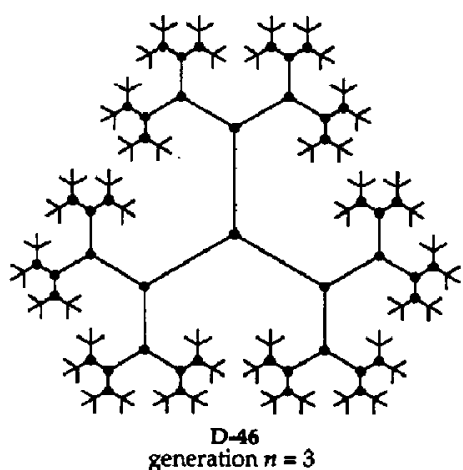

generation $n=3$

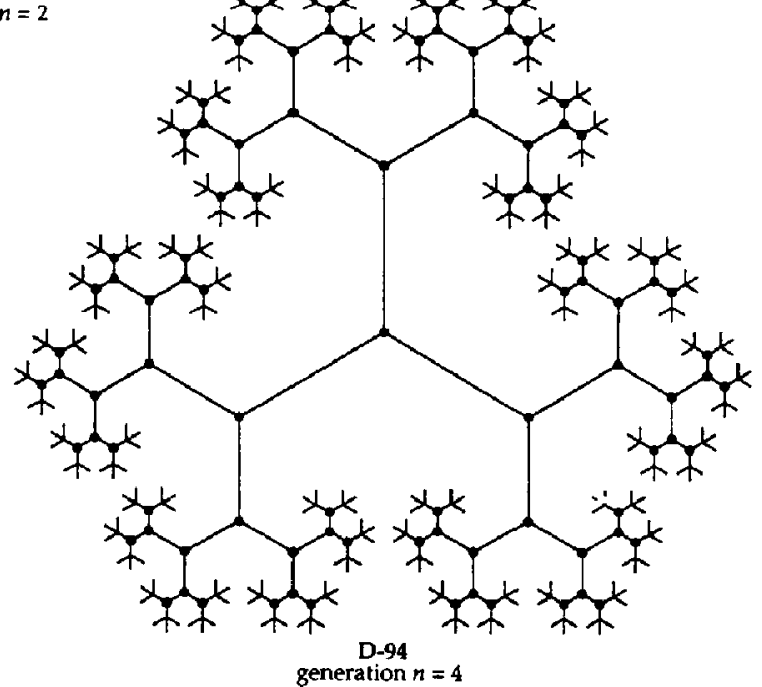

Figure 1. Graphical representation of molecular structures of the studied dendrimers.

thermally labile species. However, mass spectrometric characterization of these compounds is complicated by difficulties in forming intact molecular ions. To accomplish this, it was necessary to develop new procedures that permit use of the unique analytical capabilities of MALDI and laser desorption-chemical ionization (LDCl). Because both are soft ionization techniques, they are well suited for the present application. The only previous report of a hydrocarbon polymer analysis by MALDI is found in a recent article by Bahr et al. [2] in which MALDI analysis of a polystyrene sample is discussed. Also recently, the use of laser desorption-

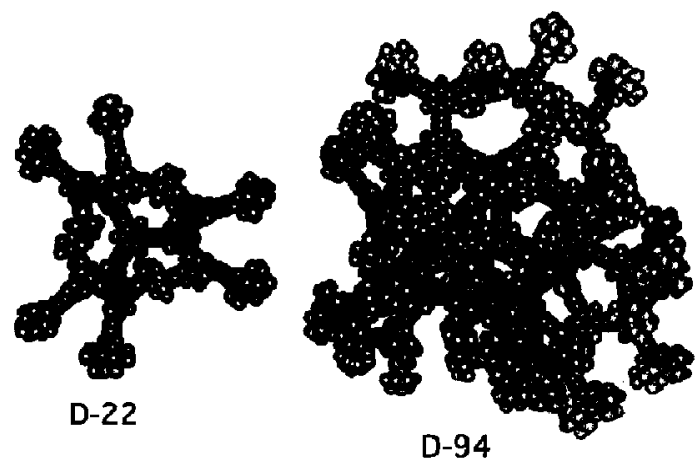

Figure 2. Space-filling madels of the D-22 and D-94 dendrimers. silver ion chemical ionization (CI) was demonstrated as a reasonably general method for analysis of hydrocarbon polymers. This FTMS method was applied [3] to a variety of polymers with masses as great as 8000 u.

However, analyses of the phenyl acetylene dendrimers, even by MALDI, proved challenging because they are soluble only in nonpolar solvents. Nevertheless, it is possible to accomplish mass spectral analysis by using a variety of techniques, including LD-CI, matrix-assisted laser desorption-chemical ionization (MALD-CI) and MALDI. LD-CI FTMS is applied to dendrimers ranging in mass up to $3600 \mathrm{u}$. MALD-CI is developed and used with FTMS analysis of dendrimers ranging up to $15 \mathrm{ku}$ by combining a recently introduced UV-absorbing matrix [4] with silver CI. MALDI TOF is successful for all dendrimers by using a new matrix, retinoic acid, which is soluble in nonpolar solvents.

\section{Experimental}

\section{Time-of-Flight Mass Spectrometry}

Sample Preparation. A $0.034-\mathrm{M}$ retinoic acid matrix solution was prepared by dissolving all trans-retinoic acid (Sigma Chemical Company, St. Louis, MO) in methylene chloride. Prior to its use, the solution was irradiated with fluorescent light for approximately 48 
$h$ at room temperature (see Results and Discussion for the rationale). All dendrimer samples were dissolved in methylene chloride (Fisher Scientific, Pittsburgh, PA, ACS grade).

The D-4 dendrimer and retinoic acid were combined to form a solution with a matrix-analyte ratio of 5. For purposes of mass calibration, a 1.8- $\mu \mathrm{L}$ aliquot of a $0.02-\mathrm{M}$ solution of poly(ethylene glycol)-2000 (PEG 2000) was deposited and allowed to dry on the stainless steel laser target prior to deposition of an aliquot of the matrix-D-4 mixture containing $240 \mathrm{pmol}$ of the dendrimer. Similarly, dendrimer D-10 was prepared for analysis by using solutions with a matrix-analyte ratio of 15 and PEG-3000 as calibrant. For dendrimer D-22, a matrix-analyte ratio of 400 was used with PEG-2000 as calibrant. In each of the latter two cases, aliquots containing $140 \mathrm{pmol}$ of dendrimer were analyzed by using the same procedure described for the D-4 dendrimer.

Analysis of the D-46 and D-94 dendrimers was accomplished by combining retinoic acid and dendrimer samples to form methylene chloride solutions with matrix-analyte ratios of 570:1 (D-46) and 550:1 (D-94). Aliquots corresponding to 51 (D-46) and 74 (D-94) pmol of dendrimer were transferred to the stainless steel laser target for analysis. In each case, for calibration, $1-\mu \mathrm{L}$ aliquots of a $0.02-\mathrm{M}$ solution of PEG calibrant was deposited and allowed to dry on the laser target prior to addition of the dendrimer-matrix mixture. For D-46, the calibrant was PEG-3000 and for D-94, the calibrant was PEG-1000.

TOF Instrumentation. MALDI time-of-flight mass spectra were measured with a LaserTec research mass spectrometer (Vestec Corporation, Houston, TX) equipped with a neodymium-YAG laser (Lumonics YAGMASTER 200, Lumonics Ltd., Warwickeshire, England). All samples were desorbed by using the frequency-tripled (355-nm) output of the laser. Sample irradiance was controlled with a variable attenuator (Model 935-5-OPT UV, Newport Research Corporation, (Irvine) CA) and focused to illuminate an average spot size of diameter $20 \mu \mathrm{m}$.

All spectra were measured by using the linear timeof-flight mode (1.2-m flight path). Ions were accelerated with a potential of $29 \mathrm{kV}$ and detected by an ion multiplier biased at $5 \mathrm{kV}$ except for $\mathrm{D}-\mathbf{1 0}$ dendrimer spectra, where the multiplier was held at $3.5 \mathrm{kV}$. The resulting ion current was monitored at 2-ns intervals by using a digital oscilloscope (Model TDS 520, Tck tronix Inc., Beaverton, OR). Mass spectra were generated from the average of 7 to 23 single acquisitions and the resulting spectra were smoothed with a fivc-point Savitsky-Golay smooth.

Mass assignments were prepared by estimating the centroid of the peaks with a commercial software package (Grams 386, Galactic Industries Corporation, Salem, NH). All dendrimer mass spectra were calibrated by using sodium-attached ions of the appropriate PEG calibrant as an internal reference.

\section{Fourier Transform Mass Spectrometry}

Sample Preparation. All dendrimer samples were dissolved in methylene chloride to form $5.0 \times 10^{-4}-\mathrm{M}$ solutions. A $0.05-\mathrm{M}$ solution of the UV-absorbing matrix 1,4-diphenyl-1,3-butadiene (Aldrich Chemical Co. Milwaukee, WI) in methylene chloride was used for matrix-assisted laser desorption-chemical ionization experiments. Sample solutions were applied to the laser target using an aerosol nebulizer. For direct laser desorption (D-4, D-10) the sample was then placed in the mass spectrometer and analyzed. For silver nitrate LD-CI, $50 \mu \mathrm{L}$ of a silver nitrate (Fisher Scientific, Pittsburgh, PA) saturated ethanol (Quantum Chemical Corp., Tuscola, IL) solution was first sprayed onto a rotating stainless steel probe tip and allowed to dry. Next, $300 \mu \mathrm{L}$ of the appropriate $5.0 \times 10^{-4}-\mathrm{M}$ dendrimer solution (D-4, D-10, or D-22) was sprayed on top of the silver nitrate and allowed to dry. For MALD-CI, $500 \mu \mathrm{L}$ of the matrix solution and either $100 \mu \mathrm{L}$ of the D-46 solution or $50 \mu \mathrm{L}$ of the D-94 solution, together with $20 \mu \mathrm{L}$ of the saturated silver nitrate solution and $200 \mu \mathrm{L}$ of pure methanol, were mixed together. Matrix -analyte molar ratios were thus 500:1 (D-46) and 1000:1 (D-94). For analysis, each mixture was sprayed onto a rotating stainless steel probe tip and allowed to dry. Following deposition, samples were introduced into the vacuum system and the system was allowed to pump down to a pressure of $\sim 5 \times 10^{-8}$ torr for LD-CI and $\sim 5 \times 10^{-7}$ torr for MALD-CI prior to spectral data acquisition.

Instrumentation. FTMS studies were performed using an Extrel FTMS-2000 Fourier transform mass spectrometer (Millipore Corporation, Madison, WI) equipped with a 7-T superconducting magnet, differentially pumped source and analyzer regions separated by a 2-mm conductance limit, and an automatic solids probe. A Tachisto 215 pulsed $\mathrm{CO}_{2}$ laser (Tachisto Laser Systems, Inc. Needham, MA) was used for laser desorption and laser desorption-chemical ionization experiments. The laser was operated at $10.6 \mu \mathrm{m}$ and focused to obtain power densities between $10^{6}$ and $10^{8}$ $\mathrm{W} / \mathrm{cm}^{2}$ with a $80 \pm 40$-ns pulse width. For matrix-assisted laser desorption-chemical ionization, the 357-nm output of a Lambda Physik FL-2001 (Göttingen, Germany) dye laser was used. The beam was focused to power densities of between $10^{6}$ and $10^{7} \mathrm{~W} / \mathrm{cm}^{2}$ with a 28-ns pulse width. The dye laser [with a $0.6-\mathrm{g} / \mathrm{L}$ dioxane solution of 2,2 $2^{\prime \prime \prime}$-dimethyl-p-quaterphenyl (BMQ, Lambda Physik)] was pumped with the 308-nm output of a Lambda Physik EMG 201-MSC excimer laser.

Spectral Measurement Conditions. D-4 and D-10 spectra were obtained by using analyzer-cell receive experiments with $200-$ and $700-\mu$ s transfer times, respectively. Attempts to transfer the D-22, D-46, and D-94 analyte ions to the analyzer cell were unsuccessful. Therefore, their spectra were measured for ions trapped 
in the source cell. The D-46 and D-94 spectra were obtained with MALD-CI, which requires the use of a retardation potential to sluw the entergetic desurbed ions and allow trapping at normal potentials [5]. Furthermore, the optimum length of the retardation period is a function of the mass of the analyte [6]. Accordingly, a 9.75-V retarding potential was applied for either $120 \mu \mathrm{s}$ (D-46) or $200 \mu \mathrm{s}$ (D-94) prior to application of analysis trapping potentials. Spectral data acquisition was performed in direct mode with bandwidths and number of data points chosen based upon resolution and signal-to-noise considerations. Relevant spectral parameters are summarized in Table 1.

Data Analysis. Data were baseline-corrected and augmented with an equal number of zeros before Fourier transformation. All spectra were postcalibrated with poly(ethylene glycol)-1000 spectra obtained within $1 \mathrm{~h}$ by using the same trapping procedures and potentials as for the corresponding dendrimer spectra. Peak mass assignments were determined with a Lorentzian fit to the data points of the spectrum (not interpolated). For D-4, D-10, and D-22 dendrimers, where isotopic patterns were resolved, the mass accuracy was calculated by comparing the measured mass at each isotopic peak with the theoretical mass of that peak's isotopic composition. For the D-46 and D-94 compounds, where the isotopic pattern was not resolved, the average molecular weight for the predicted structure was compared with the mass corresponding to the centroid of the unresolved isotopic multiplet.

\section{Results and Discussion}

As mentioned earlier, laser desorption mass spectrometry of hydrocarbon polymers and dendrimers is somewhat more difficult than for their heteroatom-containing counterparts. It is therefore of interest to explore a variety of approaches to that analytical task. Another motivation of the present study is the need to obtain experimental evidence that the synthetic dendrimers investigated have mass spectral properties in accord with their putative structures. From the data presented

Table 1. Spectral data acquisition parameters for FTMS spectra of dendrimers

\begin{tabular}{lcccc}
\hline Dendrimer & $V_{\text {traplvalts) }}$ & Delay (s) & $\begin{array}{c}\text { Observation } \\
\text { bandwidth } \\
\text { (kHz) }\end{array}$ & $\begin{array}{c}\text { Number } \\
\text { of data } \\
\text { points }\end{array}$ \\
\hline \hline D-4 & 0.2 & $1-5$ & 200 & 262,144 \\
D-10 & 0.2 & $1-5$ & 100 & 131,072 \\
D-22 & 0.5 & $1-5$ & 30 & 131,072 \\
D-46 & 1.0 & $1-5$ & 200 & 65,536 \\
D-94 & 1.0 & $1.5 \times 10^{-3}$ & 200 & 32,768 \\
\hline
\end{tabular}

aelay time between laser desorption pulse and spectral data acquisition. Because of very short trapping lifetimes for D-94 ions. maximum delay times were no longer than tens of milliseconds. here, it is clear that MALDI TOF and LD FTMS are complementary tools and, together, provide additional convincing evidence of the success of the convergent synthetic strategy described elsewhere [1]. For convenience, the MALDI TOF and FTMS results will be discussed separately.

\section{MALDI TOF}

TOF Spectra. MALDI TOF spectra of the five dendrimers studied appear in Figures 3-7 and the corre sponding mass accuracy and resolution data are summarized in Table 2. It is seen from these data that the resolving power (as estimated by measuring peak widths at half height) averages about 900 . and mass measurement errors range between 0.002 and $0.017 \%$.

In the least accurate of the molecular ion measurements, seen in the D-4 spectrum, mass accuracy of $0.017 \%$ is found for the molecular ion, which is measured with resolving power of about 700 . For this compound, only low matrix-analyte ratios yield spectra with high signal-to-noise ratios. Thus, this spectrum was obtained by using a matrix-analyte ratio of 5:1. Other D-4 spectra were obtained with matrix-analyte ratios as low as 1:1. Although these are unusually low matrix-analyte ratios for MALDI, they are not unprecedented. For example, Hutchens and Yip [7] demonstrated MALDI by using stoichiometric matrix-analyte ratios. It is also clear that these are true MALDI spectra, because no dendrimer ions are detected if samples are irradiated with 355-nm light in the absence of matrix. It is interesting that the spectrum in Figure 3 shows the probable presence of higher mass cluster ions, although overlap of the PEG-2000 internal calibrant spectrum makes it difficult to see this unambiguously. This spectrum was measured at laser fluences just above those that induce the first appearance of matrix ions, yet the presence of clusters is indicated. At higher laser fluences, higher mass clus-

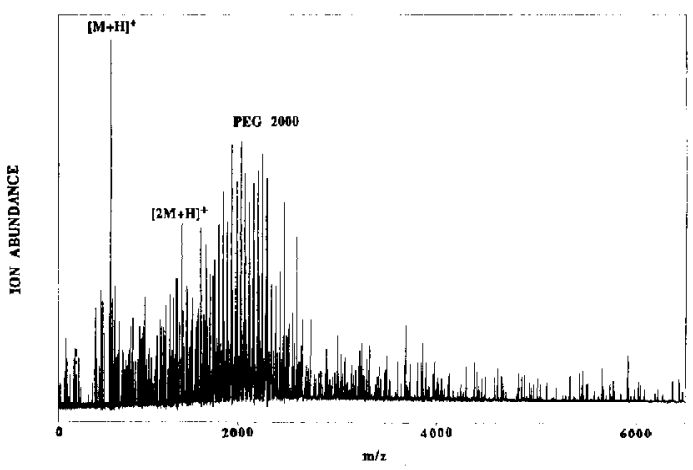

Figure 3. MALDI TOF mass spectrum of 3-cascade:benzene [31,3,5]:5-ethynyl-1,3-di(tert-butyl) benzene, that contains four phenyl acetylene monomer units (D-4). The spectrum was measured by using retinoic acid as the matrix and poly(ethylene glycol) as an internal mass calibrant. 


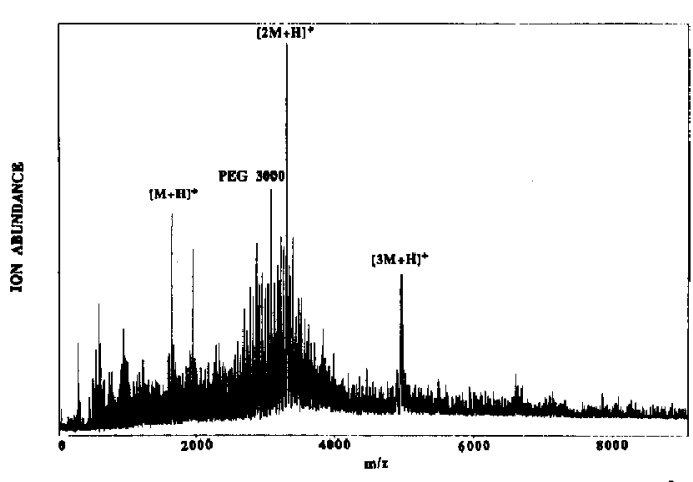

Figure 4. MALDI TOF mass spectrum of 6-cascadebenzene [31,3,5]:(5-ethynyl-1,3-phenylene):5-ethynyl-1,3-di(tert-butyl) benzene that contains 10 phenyl acetylene monomer units (D-10). The spectrum was measured by using retinoic acid as the matrix and poly(ethylene glycol) as an internal mass calibrant.

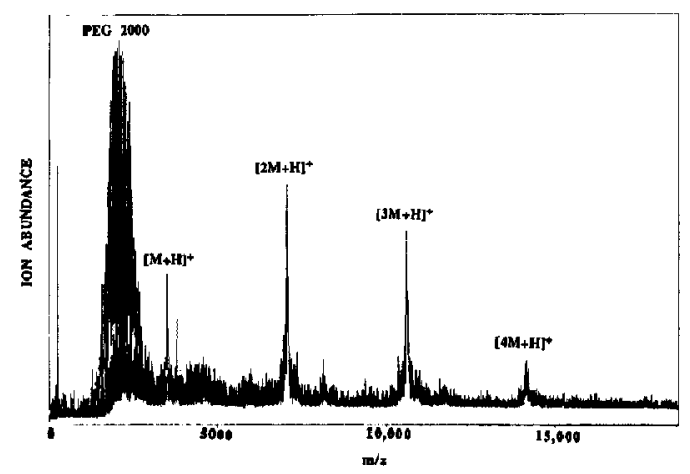

Figure 5. MALDI TOF mass spectrum of 12-cascade:benzene [3-1,3,5]:(5-ethynyl-1,3-phenylene):5-ethynyl-1,3-di(tert-butyl) benzene that contains 22 phenyl acetylene monomer units (D-22). The spectrum was measured by using retinoic acid as the matrix and poly(ethylene glycol) as an internal mass calibrant.

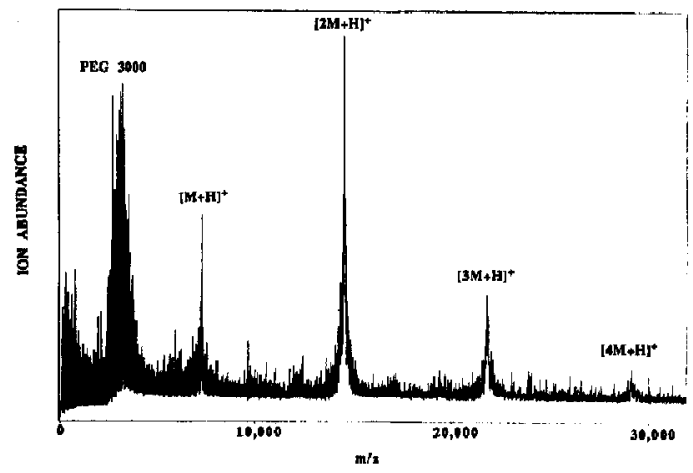

Figure 6. MALDI TOF mass spectrum of 24-cascade:benzene [3-1,3,5]:(5-ethynyl-1,3-phenylene):5-ethynyl-1,3-di(tert-butyl) benzene that contains 46 phenyl acetylene monomer units (D-46). The spectrum was measured using retinoic acid as the matrix and poly(ethylene glycol) as an internal mass calibrant.

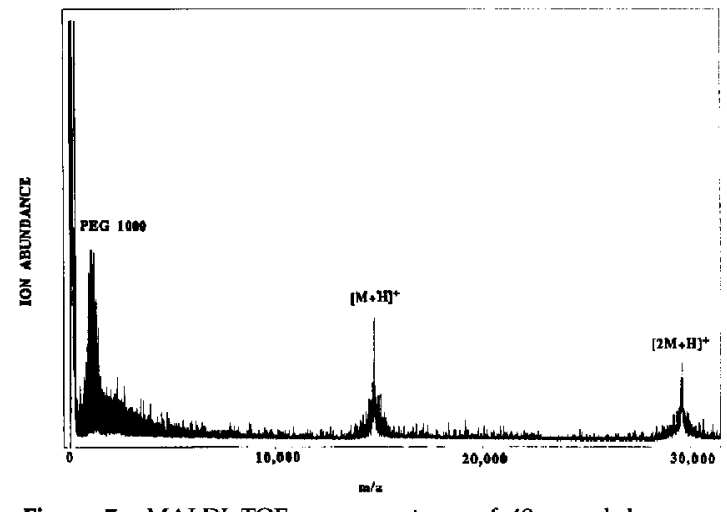

Figure 7. MALDI TOF mass spectrum of 48-cascade:benzene [3-1,3,5]:(5-ethynyl-1,3-phenylene):5-ethynyl-1,3-di(tert-butyl) benzene, that contains 94 phenyl acetylene monomer units (D-94). The spectrum was measured using retinoic acid as the matrix and poly(ethylene glycol) as an internal mass calibrant.

ters become more abundant. The large number of minor peaks along the baseline of this spectrum are suspected to result from fragments of PEG and the higher mass dendrimer clusters. Spectra obtained in the absence of PEG show fewer fragment ions. As Figure 4 shows, D-10 spectra measured under similar conditions as the D-4 show abundant molecular ions in addition to peaks corresponding to dimer and trimer clusters.

The D-22 spectrum (Figure 5) was measured using a matrix-analyte ratio of approximately $400: 1$. Here too, in addition to the expected molecular ion, the presence of clusters up to a tetrameric species is evident and the dimer and trimer clusters are more abundant than the molecular ion species. A relatively abundant matrix adduct $(\mathrm{m} / z$ 3834.2, not labeled in the spectrum) of the molecular ion is seen. Such adducts are also detected for the dimer and trimer species. There is also much less evidence of fragmentation than seen with the lower mass dendrimers, as indicated by low abundance ion signals along the baseline. This observation is consistent with the expected moderating effect of a higher matrix-analyte ratio.

The D-46 spectrum, obtained using PEG-3000 as internal calibrant, is shown in Figure 6. As is the case for D-22, the dimer cluster is the most abundant dendrimer ion detected and is about two times the abun-

Table 2. UV MALDI time-of-flight mass spectrometry results

\begin{tabular}{lrrrr}
\hline Dendrimer & $\begin{array}{c}\text { Reference } \\
\text { mass }^{*}\end{array}$ & $\begin{array}{c}\text { Measured } \\
\text { mass }\end{array}$ & $\begin{array}{c}\text { Mass error } \\
\text { (ppm) }\end{array}$ & $\begin{array}{c}\text { Resolving } \\
\text { power }\end{array}$ \\
\hline \hline D-4 & 716.18 & 716.3 & 168 & 680 \\
D-10 & 1653.48 & 1653.4 & 48 & 1048 \\
D-22 & 3528.21 & 3527.7 & 145 & 953 \\
D-46 & 7277.69 & 7277.3 & 54 & 800 \\
D-94 & 14776.57 & 14776.2 & 25 & 1100 \\
\hline
\end{tabular}

\footnotetext{
${ }^{a}$ Weighted average, $[\mathrm{M}+\mathrm{H}]^{+}$ion.
} 
dance of the molecular ion species. Initially, PEG was added to the matrix-analyte solution in the ratio $80: 1$ with the analyte. This resulted in spectra that were still well resolved, but it was difficult to detect D-46 ions and many fragments and adducts were present. It was suspected that cocrystallization of PEG with the matrix-analyte mixture was the cause of the low signals for the D-46. Accordingly, 0.02-M solution of PEG-3000 was deposited onto the surface of the probe tip, dryed, and then the matrix-analyte solution deposited on top of the treated surface. With this procedure, it is possible to obtain substantially better signals for the analyte ions while maintaining a signal-to-noise ratio in excess of 20:1 for the most abundant PEG calibrant ions.

Figure 7, which is the D-94 dendrimer spectrum, lacks any evidence of clusters larger than the dimer, has excellent accuracy, and shows a resolving power of about 1100 .

Prompt Fragmentation under MALDI TOF Conditions. As the spectra reveal, the lower mass dendrimers and their related cluster ions are relatively susceptible to prompt fragmentation. The evidence of abundant fragment ions throughout the spectrum is not typical of spectra generated by MALDI, which is known for producing little ion fragmentation. These peaks can most likely be attributed to prompt fragmentation of both the monomer and higher mass clusters. The high degree of fragmentation of these complexes suggests that for a significant population of the dendrimers, the critical energy for decomposition has been exceeded during the MALDI process. This may very well result from the low matrix-analyte ratios used for D-4, D-10, and D-22. Interestingly, the higher mass dendrimer ions from D-46 and D-94, which should be formed with higher energies during MALDI [8] appear to be more stable. This may be the result of the combination of the effects of much higher matrix-analyte ratios and the enhanced ability of such ions to distribute internal energy resulting from the large number of vibrational degrees of freedom.

Matrix Effects. MALDI spectra acquired by using dihydroxybenzoic acid as the matrix showed very low abundances of molecular ions and evidence that the conditions favored cluster formation, with cluster ion abundances 10 to 20 times those of the molecular ion. Use of other matrices typically employed for MALDI (c.g., $\alpha$-cyano-4-hydroxy cinnamic acid or sinnapinic acid) produced either no spectra or spectra with exceptionally poor resolution and signal-to-noise ratios. For these matrices, the MALDI process was complicated by the low solubility of these matrices in nonpolar solvents, which yielded poor ionization sensitivity, possibly as a result of inadequate cocrystallization of matrix and analyte [9]. Attempts to codissolve dendrimers and these matrices in acetonitrilealcohol-water solutions were almost completely un- successful owing to the almost total insolubility of dendrimers in polar solvents.

As a consequence, a new matrix, retinoic acid was investigated. This material is highly soluble in methylene chloride and is very well suited to dendrimer analyses. As Figures $3^{-7}$ illustrate, excellent signal-tonoise ratios and resolution are obtained with this matrix. In general, spectra with higher signal-to-noise ratios were obtained when the dendrimer-matrix solution was analyzed without PEG calibrant present. This problem was minimized by deposition of the sample and calibrant in layers rather than mixing the analyte and internal standard. In the presence of PEG, sample deposition of at least $90 \mathrm{pmol}$ of a dendrimer on the laser target is required to obtain dendrimer spectra with signal-to-noise ratios of 20 or greater.

It is interesting that poor spectra are obtained when all trans-retinoic acid is used directly as supplied with no exposure to light. The retinoids are known to undergo photoisomerization [9] in solution and when exposed to light. As described in the experimental section, trans-retinoic acid was exposed to fluorescent room light for at least $72 \mathrm{~h}$ prior to its use as a MALDI matrix. Subsequently, pure 13-cis-retinoic acid was used as a MALDI matrix for analysis of a sample of the D-46 dendrimer (in the absence of PEG) and gave a spectrum with a signal-to-noise ratio $(\mathrm{S} / \mathrm{NJ})$ greater than 20:1 and resolving power greater than 1000 with 10 pmol deposited. With this matrix, the D-46 dendrimer was detectable with $S / N \geq 3: 1$ when 1 pmol was deposited. However, subsequent experiments show that trans-retinoic acid exposed to room light generally gives equally good results, and that there is no need to use the more expensive $c$ is isomer as a MALDI matrix.

Clusters. Spectral peaks at masses higher than that of the molecular ion appear in all the dendrimer spectra. Clusters observed for each dendrimer and their masses are summarized in Table 3. When PEG internal standards are used, some cluster ions with masses falling in the same mass range are impossible to distinguish from PEG ions. However, the clusters also can be observed in the absence of internal standards, with some sacrifice in mass measurement accuracy. Although the masses of the clusters are not determined with high accuracy, there is qualitative agreement with the interpretation that these are, in fact, dendrimer clusters. For example, at higher laser fluences the formation of these clusters becomes more pronounced. In many of the spectra it is obvious that dimer cluster ions are the most abundant. The highly branched very open architecture of the dendrimers suggests that the cluster formation may occur through an association between the branches of the dendrimers in a type of host-guest linking. A kinetic study of the cluster formation (possibly by MALDI FTMS, provided cluster lifetimes are consistent with the much longer time scale of that measurement) could give more informa- 
Table 3. Dendrimer cluster ions observed by time-of-flight mass spectrometry

\begin{tabular}{|c|c|c|c|c|}
\hline Dendrimer & Clusters $^{a}$ & $\begin{array}{l}\text { Predicted } \\
\text { mass }\end{array}$ & $\begin{array}{l}\text { Measured } \\
\text { mass }\end{array}$ & $\begin{array}{c}\text { Resolving } \\
\text { power }\end{array}$ \\
\hline \multirow[t]{2}{*}{$\overline{D-4}$} & {$[2 \mathrm{M}+\mathrm{H}]^{+}$} & 1431.4 & 1430.1 & 213 \\
\hline & {$\left[3 \mathrm{M}+\mathrm{H}^{+}\right.$} & 2146.9 & 2151.7 & - \\
\hline \multirow[t]{3}{*}{ D-10 } & {$[2 M+H]^{-1}$} & 3305.8 & 3303.5 & 274 \\
\hline & {$[3 \mathrm{M}+\mathrm{H}]^{+}$} & 4958.2 & 4957.6 & 403 \\
\hline & {$[4 \mathrm{M}+\mathrm{H}]^{+}$} & 6610.6 & 6602.9 & - \\
\hline \multirow[t]{3}{*}{ D-22 } & {$\left[2 \mathrm{M}+\mathrm{H}^{+}\right.$} & 7054.4 & 7053.4 & 233 \\
\hline & {$\left[3 M+\mathrm{HI}^{+}\right.$} & 10581.1 & 10582.5 & 196 \\
\hline & {$\left[4 M+\mathrm{H}^{+}\right.$} & 14107.8 & 14150.5 & 133 \\
\hline \multirow[t]{3}{*}{ D-46 } & {$[2 \mathrm{M}+\mathrm{H}]^{+}$} & 14553.6 & 14520.7 & 285 \\
\hline & {$[3 \mathrm{M}+\mathrm{H}]^{+}$} & 21829.9 & 21800.6 & 192 \\
\hline & {$[4 \mathrm{M}+\mathrm{H}]^{+}$} & 29106.2 & 29104.3 & 239 \\
\hline D-94 & {$[2 \mathrm{M}+\mathrm{H}]^{+}$} & 29551.4 & 29578.0 & 240 \\
\hline
\end{tabular}

${ }^{a}$ it is assumed these are proton-bound dimers, although mass resolving power does not allow that to be determined.

tion regarding the stability of the various cluster ions in the gas phase.

\section{Laser Desorption Fourier Transform Mass Spectrometry}

Infrared Laser Desorption and Silver Chemical Ionization Laser Desorption. Dendrimers D-4 and D-10 yielded molecular ions by direct laser desorption-ionization with the carbon dioxide laser. However, it was not possible to obtain dendrimer spectra for D-22, D-46, and D-94 dendrimers under conventional laser desorption conditions. Application of the silver nitrate method described earlier [3] readily yielded silver-attached molecular ions for all of the dendrimers studied.

High-resolution mass spectra were obtained for D-4, D-10, and D-22 with better than 14-ppm mass measurement accuracy for molecular ion species. These results are in good accord with the MALDI TOF measurements for these same compounds and are complementary in that these highly accurate measurements are for the dendrimers where the TOF results had the worst accuracy. Figures 8 and 9 show the molecular ion regions of D-4 and D-10 dendrimer spectra obtained with conventional laser desorption-ionization FTMS. Figure 10 shows the silver-attached molecular ion region of the D-22 LD-CI spectrum. Although spectra could be obtained for D-46 and D-94 infrared LD-CI, resolution was so poor that alternative methods were sought. Table 4 summarizes the FTMS laser desorption mass spectral results for the dendrimer analytes.

UV MALD-Silver CI. As mentioned above, although resolution was excellent for the lower mass dendrimers, it proved impossible to obtain high resolution for the higher mass D-46 and D-94. For infrared LD-CI, any delay after the laser desorption event resulted in

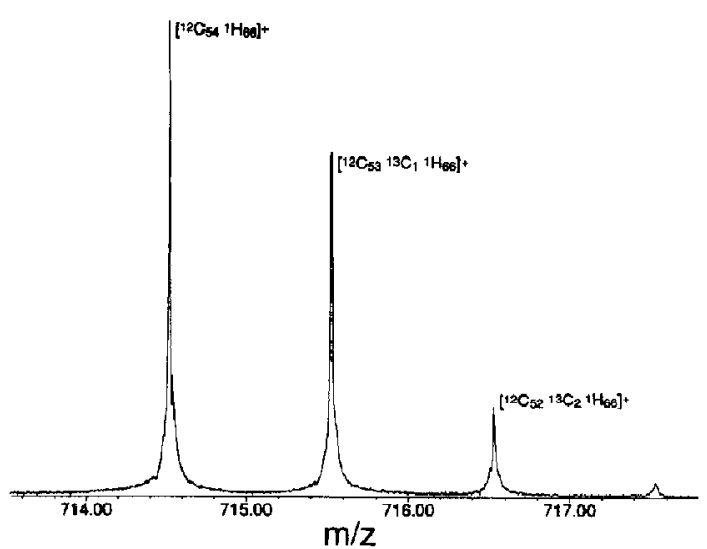

Figure 8. Infrared laser desorption Fourier transform mass spectrum for the molecular ion region of 3-cascade:benzene[31,3,5]:5-ethynyl-1,3-di(tert-butyl) benzene.

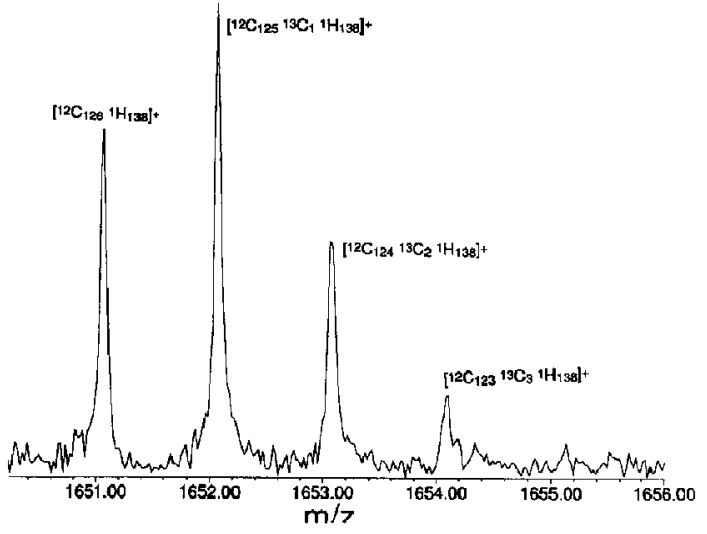

Figure 9. Infrared laser desorption Fourier transform mass spectrum for molecular ion region of 6-cascade:benzene [31,3,5]:\{5-ethynyl-1,3-phenylene):5-ethynyl-1,3-di(tert-butyl) benzene.

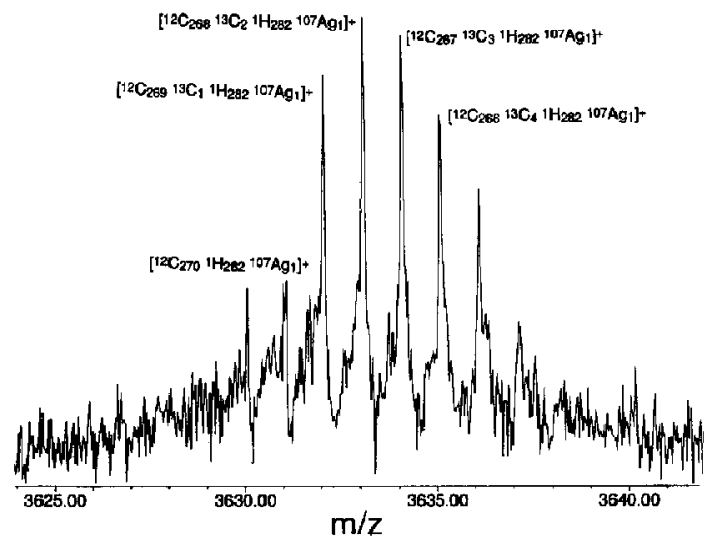

Figure 10. Infrared laser desorption Fourier transform mass spectrum for molecular ion region of 12-cascade:benzene [3-1,3,5]: (5-ethynyl-1,3-phenylene): 5-ethynyl-1,3-di(tert-butyl) benzene. 
Table 4. Fourier transform mass spectrometry results

\begin{tabular}{lccccc}
\hline Dendrimer & $\begin{array}{c}\text { Reference } \\
\text { mass }\end{array}$ & $\begin{array}{c}\text { Measured } \\
\text { mass }\end{array}$ & $\begin{array}{c}\text { Mass error } \\
\text { (ppm) }\end{array}$ & $\begin{array}{c}\text { Resolving } \\
\text { puwer }\end{array}$ & Techluique \\
\hline \hline D-4 & $714.5164^{\mathrm{a}}$ & 714.5237 & 10 & 67,277 & LD \\
D-10 & $1651.080^{\text {b }}$ & 1651.064 & 10 & 23,305 & LD \\
D-22 & $3632.1150^{\mathrm{c}}$ & 3632.063 & 14 & 26,665 & LDCI \\
D-46 & $7384.6326^{\mathrm{d}}$ & 7384.89 & 35 & 660 & MALD-Cl \\
D-94 & $14883.6457^{\mathrm{a}}$ & 14873.4 & 688 & 248 & MALD-CI \\
\hline
\end{tabular}

a Molecular mass of ${ }^{12} \mathrm{C}_{54}{ }^{1} \mathrm{H}_{86}$

Dolecular mass of ${ }^{12} \mathrm{C}_{126} \mathrm{H}_{138^{+}}$

Molecular mass of ${ }^{12} \mathrm{C}_{209}^{126}{ }_{13} \mathrm{C}_{1} \mathrm{H}_{282}{ }^{107} \mathrm{Ag}^{+}$

${ }^{d}$ Weighted average $\mathrm{C}_{558} \mathrm{H}_{570} \mathrm{Ag}_{1}^{+}$

- Weighted average $\mathrm{C}_{1134} \mathrm{H}_{1146} \mathrm{Ag}_{1}^{+}$

serious signal loss. This precluded observation periods sufficiently long to obtain high resolution. It is likely that the rapid signal damping is due to a combination of inefficient trapping and metastable decay of these higher mass analyte ions. This is in distinct contrast to the time-of-flight MALDI observations, which produced the best accuracy and resolution and the least evidence of fragmentation for these same compounds. Accordingly, it was decided to attempt to take advantage of the soft desorption-ionization characteristics of MALDI [10, 11]. Most of the matrices developed to date have targeted proteins and other polar analytes, and are thus not effective for nonpolar analytes such as the dendrimers. However, in a recent article Cornett et al. [12] reported that the nonpolar molecule dipheny] butadiene is an effective matrix. Diphenyl butadiene is soluble in nonpolar solvents and absorbs strongly at convenient laser wavelengths ( $357 \mathrm{~nm}$ in this case). Its application in conjunction with silver nitrate produces D-46 ion populations that are stable for long time periods (seconds), which allows improved resolution for both the D-46 and D-94 to be measured (Figures 11 and 12). Even 5o, resolution for these two compounds is still worse than that obtained by MALDI TOF. It appears that metastable decay, even under MALD

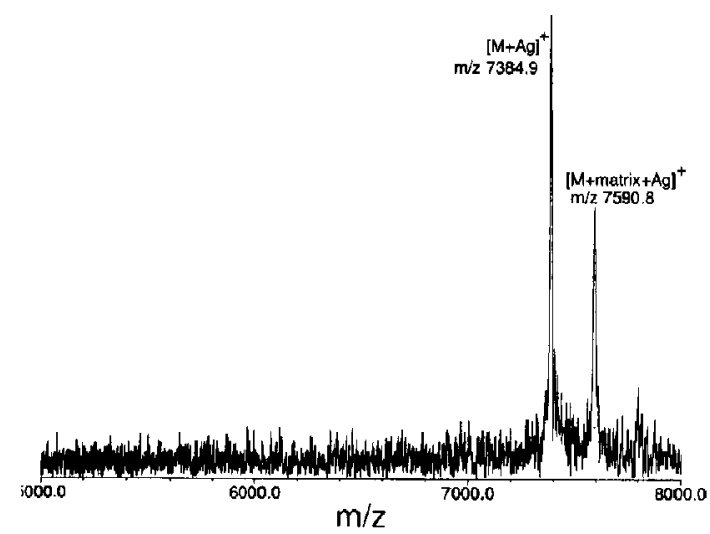

Figure 11. UV MALD-CI Fourier transform partial mass spectrum of 24-cascade:benzene [3-1,3,5]:(5-ethynyl-1,3-phenylene):5ethynyl-1,3-di(tert-butyl)benzene. The two peaks correspond to the silver-attached molecular ion and its matrix adduct. conditions is still a significant problem for the D-94 dendrimer. This decay might be eliminated or reduced by further optimization of experimental parameters. However, optimization of experimental conditions for the D-94 analysis was not pursued because resolution for the higher dendrimers is currently pressure-limited because of the use of the volatile diphenyl butadiene matrix. As a consequence of this volatility, the highest quality mass spectra were obtained when the sample was first introduced into the vacuum system at relatively high pressure, that is, mid $10^{-7}$ torr. Spectral quality degraded as the system pumped down into the $10^{-8}$-torr pressure range, probably because the matrix-analyte ratio changes as the volatile matrix sublimes, leaving the nonvolatile analyte behind. Thus, although MALD-CI improved the resolution, the volatility of the matrix precluded the expected significant improvement in mass resolution.

Alternatives for Improved Mass Spectral Resolution of High Mass Dendrimers. Transfer of ions generated in the high pressure source cell to the low pressure analyzer cell would allow higher mass resolution to be obtained. However, as mentioned above, all attempts to

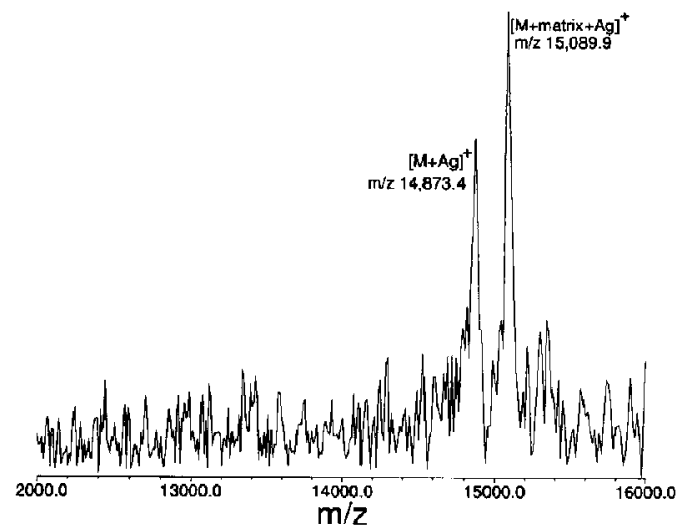

Figure 12. UV MALD-CI Fourier transform partial mass spectrum of 48-cascade:benzene [3-1,3,5]:(5-ethynyl-1,3-phenylene):5ethynyl-1,3-di(tert-butyl)benzene. The two peaks correspond to the silver-attached molecular ion and its matrix adduct. 
transfer the D-22, D-46, and D-94 dendrimers to the analyzer cell were unsuccessful. Difficulties in transferring large analytes across a conductance limit have been reported previously. In previous MALDI FTMS studies, Castoro and Wilkins [6] were unable to transfer analytes with masses greater than a few thousand mass units through the conductance limit. In their experiments, it was possible to obtain high source cell resolution because a nonvolatile MALDI matrix $(2,5-$ dihydroxybenzoic acid) was used [6]. Guan et al. [13] have employed ion axialization with a collision gas to cool ions and reduce the ion cloud radius to allow successful transfer of ions across the conductance limit. To achieve high resolution for the larger dendrimers, either a new nonvolatile matrix appropriate for MALDI FTMS must be discovered or ion transfer to the low pressure region of the analyzer cell can be accomplished via the axialization method. Such studies are currently being pursued and hold some promise for improved high mass dendrimer analysis in the future.

\section{Conclusion}

Analysis of the phenyl acetylene dendrimers by mass spectrometry provides unique challenges. Because most of the these hydrocarbon dendrimers are nonvolatile and non-polar, standard MALDI methods proved to be inapplicable. It was therefore necessary to develop a number of new analytical approaches that allowed accurate mass measurements of molecular ion species for the five dendrimers analyzed. In addition to development of a ncw matrix suitable for samples of this type, it was also possible to adapt the relatively new method of laser desorption silver chemical ionization by FTMS to the present application. These results establish the promise of these approaches for future similar analytical applications. We intend to explore further high resolution FTMS of the higher mass dendrimers by use of the retinoic acid matrix and by possible application of axialization methods to extend spectral observation times.

\section{Acknowledgments}

JSM gratefully acknowledges support from the National Science Foundation under grant DMR-92-12054. CLW acknowledges National Science Foundation support under grant CHE-92-01277 and a gift from the W. M. Keck Foundation. We thank Professor Everly Fleischer for producing Figure 2 and Professor Curtis Monnig for helpful discussions.

\section{References}

1. Xu, Z.; Kahr, M.; Walker, K. L.; Wilkins, C. L.; Moore, J. S. J. Am. Chem. Soc., in press.

2. Bahr, U.; Deppe, A.; Karas, M.; Hillenkamp, F.; Giessman, U. Anal. Chem. 1992, 64, 2866.

3. Kahr, M. S.; Wilkins, C. L. J. Am. Soc. Mass Spectrom. 1993, 6, 453.

4. Comett, D. S.; Duncan, M. A.; Amster, I. J. Anal. Chem. 1993, $65,1753$.

5. Castoro, J. A.; Köster, C.; Wilkins, C. L. Rapid Commun. Mass Spectrom. 1992, 6, 239.

6. Castoro, J. A.; Wilkins, C. L. Anal. Chem. 1993, 65, 2621.

7. Hutchens, T. W.; Yip, T. T. Rapid Commun. Mass Spectrom. 1993, 7, 576.

8. Beavis, R. C,; Chait, B. T. Chem. Phys. Lett. 1991, 2, 479.

9. Ehring, H.; Karas, M.; Hillenkamp, F. Org. Mass Spectrom. 1992, 27, 472 .

10. Karas, M.; Hillenkamp, F. Anal. Chem. 1988, 60, 2301.

11. Tanaka, K.; Waki, H.; Ido, Y.; Akita, S.; Yoshida, Y.; Yoshida, T. Rapid Commun. Mass Spectrom. 1988, 2, 151.

12. Comett, D. S.; Duncan, M. A.; Amster, I. J. Anal. Chem. 1993, $65,2608$.

13. Guan, S.; Wahl, M. C.; Wood, T. D.; Marshall, A. G. Anal. Chem. 1993, 65, 1753. 